\title{
SMACK: Decoupling Source Language Details from Verifier Implementations ${ }^{\star}$
}

\author{
Zvonimir Rakamarić ${ }^{1}$ and Michael Emmi ${ }^{2}$ \\ 1 School of Computing, University of Utah, USA \\ zvonimir@cs.utah.edu \\ 2 IMDEA Software Institute, Spain \\ michael.emmi@imdea.org
}

\begin{abstract}
A major obstacle to putting software verification research into practice is the high cost of developing the infrastructure enabling the application of verification algorithms to actual production code, in all of its complexity. Handling an entire programming language is a huge endeavor that few researchers are willing to undertake; even fewer could invest the effort to implement a verification algorithm for many source languages. To decouple the implementations of verification algorithms from the details of source languages, and enable rapid prototyping on production code, we have developed SMACK. At its core, SMACK is a translator from the LLVM intermediate representation (IR) into the Boogie intermediate verification language (IVL). Sourcing LLVM exploits an increasing number of compiler front ends, optimizations, and analyses. Targeting Boogie exploits a canonical platform which simplifies the implementation of algorithms for verification, model checking, and abstract interpretation. Our initial experience in verifying C-language programs is encouraging: SMACK is competitive in SV-COMP benchmarks, is able to translate large programs (100 KLOC), and is being used in several verification research prototypes.
\end{abstract}

\section{Introduction}

A major obstacle to putting software verification research into practice is the high cost of developing the infrastructure enabling the application of verification algorithms to actual production code, in all of its complexity. Each high-level programming language brings a diverse assortment of statements and expressions with varying semantics. Handling an entire language is a huge effort which few researchers are willing to undertake; even fewer could invest the effort required to implement their verification algorithms for multiple source languages.

To address this problem, we introduce SMACK: a translator from the LLVM compiler's popular intermediate representation (IR) 2724 into the Boogie intermediate verification language (IVL) 1926. SMACK's primary function is to precisely and efficiently translate the rich set of LLVM-IR features, including dynamic memory allocation and pointer arithmetic, to the comparatively-simple

* Partially supported by NSF award CCF 1346756.

A. Biere and R. Bloem (Eds.): CAV 2014, LNCS 8559, pp. 106-113, 2014.

(C) Springer International Publishing Switzerland 2014 
Boogie IVL, which does not include such features. SMACK thus promotes the development of verification algorithms on simple IVLs, effectively decoupling the implementations of verification algorithms from the details of source languages, and enabling rapid prototyping on production code. Sourcing LLVM IR exploits a rapidly-growing frontier of LLVM frontends, encompassing a diverse set of languages including $\mathrm{C} / \mathrm{C}++$, Java, Haskell, Erlang, Python, Ruby, Ada, and Fortran. In addition, SMACK benefits from code simplifications made by LLVM's optimizer, including constant propagation and dead-code elimination, as well as readily-available analyses, including LLVM's pointer analyses. Targeting Boogie IVL exploits a canonical platform which simplifies the implementation of verification algorithms due to Boogie's minimal syntax and mathematically-focused expression language, which is easily rendered into the satisfiability modulo theories (SMT) format of automated theorem provers [6]. By embracing Boogie IVL as a canonical program representation, SMACK not only simplifies the development of program verification technology, but also fosters the development of interoperable technology in which verification backends can be easily swapped.

Our initial experience in verifying C-language programs with SMACK, using Microsoft Research's Boogie and Corral [23] as backends, is encouraging. SMACK has eased the development of our research prototypes by enabling IVLlevel, rather than C-level or LLVM-level, implementations. In doing so, it appears that our approach does not significantly compromise performance, as SMACK (with Boogie and Corral backends) is competitive on SV-COMP 33 benchmarks. Furthermore, SMACK translates large, full-featured programs - including the entire Contiki operating system [15], at around $100 \mathrm{KLOC}$ of C code - and has been used on intricate implementations which make extensive use of features such as dynamic memory allocation.

While our experience with SMACK has thus far been centered on SMT-based bounded verification, i.e., validation of program assertions up to recursion-depth and loop-unroll bounds, our prior experience [1030] suggests that SMACK can also be applied straightforwardly to deductive verification, i.e., validation of assertions in programs adequately annotated with loop invariants and procedure pre- and post-conditions. While in theory SMACK is equally applicable for fully automatic unbounded verification methods (e.g., based on computing fixed points), in practice such applications may require powerful reasoning engines capable of generating quantified invariants over the unbounded maps which SMACK uses to model dynamically-allocated memory; it remains to be seen whether such applications are feasible.

SMACK is an open source project available on GitHulb implemented in roughly $4 \mathrm{~K}$ lines of $\mathrm{C}++$ code, and is integrated into the rise4fun website Currently, SMACK is supported on Linux, OSX, and Windows, and is used in several projects, including Microsoft Research's Q program verifier 3

\footnotetext{
${ }^{1}$ http://github.com/smackers/smack

2 http://rise4fun.com/SMACK

3 http://research.microsoft.com/en-us/projects/verifierq
} 


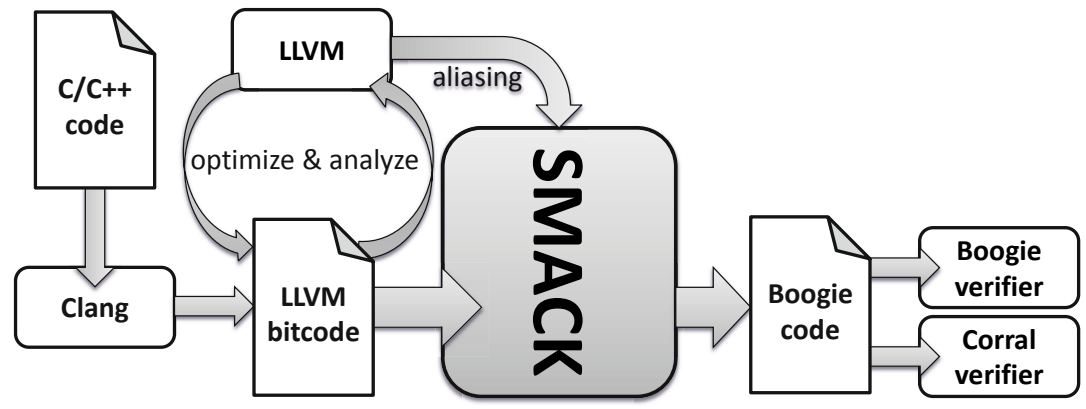

Fig. 1. Design of the SMACK modular software verification ecosystem

Related Work. Automatic verification using automated theorem provers, and in particular SMT solvers, is an active area of research. Many tools are available with various capabilities, features, and trade-offs, including Caduceus 21, Calysto 4], Cascade [34, CBMC [13, CPAchecker 17, ESBMC [16], Frama-C [17, GraVy [2, HAVOC [10, Joogie [3, KLEE [9], LLBMC [28, SATABS 12, Symbolic PathFinder 29, TASS 32, UFO 1, and VCC 14. Our SMACK effort stands alone, since none of these tools combine the language independence of leveraging a popular IR with the ease of implementation provided by IVLs. Furthermore, SMACK has been designed to accommodate a diverse set of extensions, from supporting new source language features to generating alternate IVL encodings.

\section{Translation from LLVM IR to Boogie IVL}

We have developed SMACK as one essential component of the software verification ecosystem depicted in Fig. 1. Currently, the other components include the Clang compiler [11, the LLVM compiler infrastructure [27 24], and the Boogie [1926] and Corral [23] verification engines. Beginning from a program written in $\mathrm{C} / \mathrm{C}++$, we use Clang to emit LLVM bitcode in an intermediate representation (IR) used by LLVM. LLVM IR is a typed, static single assignment (SSA), and platform-independent assembly language, and an ideal representation for LLVM's code optimizer/analyzer.

Following LLVM code optimizations, such as constant propagation and dead-code elimination, SMACK translates LLVM bitcode to code in Boogie's intermediate verification language (IVL). Boogie IVL is typed, imperative, and procedural, includes a rich mathematical expression language, and is an ideal representation for program verifiers. The Boogie programs which SMACK generates are essentially control-flow graphs with very few statements - they have goto, assignment, procedure call \& return, and assume/assert statements - which manipulate global and procedure-local variables over very few types - only integers and maps from integers to integers. For the most part, SMACK's translation is tight, in the sense that LLVM data and instructions correspond closely 
to Boogie data and instructions, modulo representing fixed-width integers with mathematical integers, 4

While there are many syntactic differences between LLVM IR and Boogie IVL, a key fundamental difference which SMACK addresses is memory representation: while LLVM IR performs dynamic allocation on the memory heap, programs in Boogie IVL have only a fixed number of global variables, albeit over unbounded types including mathematical integers and maps (i.e., arrays). Although in theory the entire heap could be represented with one single map, experience indicates that this strategy is not efficient; a verifier which represents map-type variables with array-theory expressions would suffer as the map is updated across many addresses. Instead, SMACK uses static analyses in LLVM to infer a set of memory regions which are disjoint, in the sense that two distinct regions are never accessed by the same program expression; each region of the heap is then given its own map, and each heap access translates to an expression using the accessed region's map [31. SMACK's modular design facilitates the implementation of alternate memory models by, for example, redefining: (1) the Boogie-code implementations of malloc and free to describe alternate allocation policies (which does not require recompiling SMACK), or (2) the translation of load and store operations to model heap accesses at byte-sized granularity (currently requires recompilation).

SMACK passes the resulting Boogie-IVL program to either the Boogie or Corral verifier; both function by generating verification conditions [5] which are discharged using satisfiability modulo theories (SMT) solvers, such as Z3 [18.

\section{An Example Translation}

We illustrate our verification workflow step-by-step on the program listed in Fig. 2. The $\mathrm{C}$ program (top left) is first compiled with Clang into the LLVM IR program shown on the right. In the process, calls to malloc in $\mathrm{C}$ are compiled into the respective invocations in the LLVM IR. Structure field accesses are compiled into a combination of getelementptr and load/store instructions, where getelementptr performs the structure field address computation that is subsequently accessed using load/store. Note that while the LLVM IR is a simple representation, it does include dynamic memory allocation, pointer arithmetic, and complex data types - none of which are included in the Boogie IVL.

From the LLVM IR program, SMACK generates the Boogie IVL program by leveraging LLVM's static data structure analysis (DSA) 25] to split memory into a set of disjoint regions so that pointers to two distinct regions can never alias 31. Each such region is then statically assigned its own map, and each memory access translates to an expression using the accessed region's map. In Fig. 2, based on the fact that DSA accurately reported that LLVM IR pointer variables $\% 5$ and $\{\% 6, \% 7\}$ cannot alias, SMACK statically introduced memory

\footnotetext{
${ }^{4}$ While our current implementation uses unbounded integers and maps thereof, in principle we could also use bit-vectors to model, e.g., 32-bit integers precisely.
} 
Table 1. Comparison of SMACK, CPAchecker, CBMC, and UFO on SV-COMP benchmarks. \#B is the number of benchmarks (both correct and buggy) in a suite. No-Reuse and Reuse correspond to two distinct memory models currently provided by SMACK. Experiments were performed on an Intel Core i7-3930K 3.20 GHz machine with $32 \mathrm{~GB}$ of memory running Ubuntu 12.04. All runtimes are in seconds.

\begin{tabular}{|c|c|c|c|c|c|c|c|c|c|}
\hline \multirow{3}{*}{$\begin{array}{l}\text { Benchmark } \\
\text { Suite }\end{array}$} & \multirow{3}{*}{$\# \mathrm{~B}$} & \multirow{3}{*}{ KLOC } & \multicolumn{4}{|c|}{ SMACK } & \multirow{2}{*}{\multicolumn{3}{|c|}{ SV-COMP 2014}} \\
\hline & & & \multicolumn{2}{|c|}{ No-Reuse } & \multicolumn{2}{|c|}{ Reuse } & & & \\
\hline & & & Boogie & Corral & Boogie & Corral & CPAchecker & $\mathrm{CBMC}$ & UFO \\
\hline locks & 13 & 2.3 & 9.1 & 9.3 & 9.0 & 9.3 & 365.1 & 1.4 & 2.9 \\
\hline ntdrivers-simpl & 10 & 18.1 & 12.3 & 85.7 & 12.3 & 86.4 & 43.5 & 4.6 & 3.4 \\
\hline
\end{tabular}

"CSeq does not support [heap-allocated memory] yet. Lifting these restrictions, and in particular supporting dynamic memory ... will require significant efforts."

In contrast, the Boogie IVL-based c2s tool was simple to implement, and has been used for the analysis of intricate C-language concurrent data structure implementations which make extensive use of dynamic memory allocation [8].

Despite the threat to performance incurred by separating backend verifiers from source languages, SMACK-based tools are competitive with state-of-theart verifiers. While a truly-meaningful comparison is difficult, since different verifiers generally provide different guarantees, Table 1 makes an attempt, comparing SMACK with 3 competitive verifiers (CPAchecker [7, CBMC [13], UFO [1]) on 2 benchmark suites from the SV-COMP 33 . annual software verification competition. Both suites contain both correct and buggy benchmarks, and all verifiers categorize them correctly: neither false positives nor negatives are reported 6

Note that since these are preliminary results mixing tools aimed at bug-finding (SMACK, CBMC) with those aimed at verification (CPAchecker, UFO), a direct comparison of runtimes is somewhat unfair. However, the table does illustrate that even though SMACK has not been optimized for SV-COMP benchmarks - thus far we have spent minimal effort in optimization - its performance is comparable to established verifiers which regularly participate in SV-COMP. As future work, we plan to expand these preliminary results with more benchmarks, and enroll SMACK in a future SV-COMP.

As expected, the current version of SMACK does have some limitations. First, integer datatypes are modeled with unbounded mathematical integers; this limitation can be lifted by leveraging Boogie's support for bit-vectors. Floating point datatypes pose a more serious challenge, as they are not widely supported by current software verifiers and automated theorem provers. Finally, SMACK currently precisely handles word-aligned memory accesses only.

\footnotetext{
${ }^{6}$ To make our results readily reproducible, we created a virtual machine profile in the Apt testbed facility containing all used tools, scripts, and benchmarks. It is available at https://www . aptlab.net/p/fmr/smack-cav2014.
} 


\section{References}

1. Albarghouthi, A., Li, Y., Gurfinkel, A., Chechik, M.: UFO: A framework for abstraction- and interpolation-based software verification. In: Madhusudan, P., Seshia, S.A. (eds.) CAV 2012. LNCS, vol. 7358, pp. 672-678. Springer, Heidelberg (2012)

2. Arlt, S., Rubio-González, C., Rümmer, P., Schäf, M., Shankar, N.: The gradual verifier. In: Badger, J.M., Rozier, K.Y. (eds.) NFM 2014. LNCS, vol. 8430, pp. 313-327. Springer, Heidelberg (2014)

3. Arlt, S., Rümmer, P., Schäf, M.: Joogie: From Java through Jimple to Boogie. In: ACM SIGPLAN International Workshop on State of the Art in Java Program Analysis (SOAP), pp. 3-8 (2013)

4. Babić, D., Hu, A.J.: Calysto: Scalable and precise extended static checking. In: International Conference on Software Engineering (ICSE), pp. 211-220 (2008)

5. Barnett, M., Leino, K.R.M.: Weakest-precondition of unstructured programs. In: ACM SIGPLAN-SIGSOFT Workshop on Program Analysis For Software Tools and Engineering (PASTE), pp. 82-87 (2005)

6. Barrett, C., Stump, A., Tinelli, C.: The SMT-LIB standard: Version 2.0. In: International Workshop on Satisfiability Modulo Theories (SMT) (2010)

7. Beyer, D., Keremoglu, M.E.: CPAchecker: A tool for configurable software verification. In: Gopalakrishnan, G., Qadeer, S. (eds.) CAV 2011. LNCS, vol. 6806, pp. 184-190. Springer, Heidelberg (2011)

8. Bouajjani, A., Emmi, M., Enea, C., Hamza, J.: Verifying concurrent programs against sequential specifications. In: Felleisen, M., Gardner, P. (eds.) ESOP 2013. LNCS, vol. 7792, pp. 290-309. Springer, Heidelberg (2013)

9. Cadar, C., Dunbar, D., Engler, D.: KLEE: Unassisted and automatic generation of high-coverage tests for complex systems programs. In: USENIX Conference on Operating Systems Design and Implementation (OSDI), pp. 209-224 (2008)

10. Chatterjee, S., Lahiri, S.K., Qadeer, S., Rakamarić, Z.: A reachability predicate for analyzing low-level software. In: Grumberg, O., Huth, M. (eds.) TACAS 2007. LNCS, vol. 4424, pp. 19-33. Springer, Heidelberg (2007)

11. Clang: A C language family frontend for LLVM, http://clang.1lvm.org

12. Clarke, E., Kroning, D., Sharygina, N., Yorav, K.: SATABS: SAT-based predicate abstraction for ANSI-C. In: Halbwachs, N., Zuck, L.D. (eds.) TACAS 2005. LNCS, vol. 3440, pp. 570-574. Springer, Heidelberg (2005)

13. Clarke, E., Kroning, D., Lerda, F.: A tool for checking ANSI-C programs. In: Jensen, K., Podelski, A. (eds.) TACAS 2004. LNCS, vol. 2988, pp. 168-176. Springer, Heidelberg (2004)

14. Cohen, E., Dahlweid, M., Hillebrand, M., Leinenbach, D., Moskal, M., Santen, T., Schulte, W., Tobies, S.: VCC: A practical system for verifying concurrent C. In: Berghofer, S., Nipkow, T., Urban, C., Wenzel, M. (eds.) TPHOLs 2009. LNCS, vol. 5674, pp. 23-42. Springer, Heidelberg (2009)

15. Contiki: The open source OS for the Internet of things, http://www. contiki-os.org

16. Cordeiro, L., Fischer, B., Marques-Silva, J.: SMT-based bounded model checking for embedded ANSI-C software. In: IEEE/ACM International Conference on $\mathrm{Au}-$ tomated Software Engineering (ASE), pp. 137-148 (2009)

17. Cuoq, P., Kirchner, F., Kosmatov, N., Prevosto, V., Signoles, J., Yakobowski, B.: Frama-C: A software analysis perspective. In: Eleftherakis, G., Hinchey, M., Holcombe, M. (eds.) SEFM 2012. LNCS, vol. 7504, pp. 233-247. Springer, Heidelberg (2012) 
18. de Moura, L., Bjørner, N.S.: Z3: An efficient SMT solver. In: Ramakrishnan, C.R., Rehof, J. (eds.) TACAS 2008. LNCS, vol. 4963, pp. 337-340. Springer, Heidelberg (2008)

19. DeLine, R., Leino, K.R.M.: BoogiePL: A typed procedural language for checking object-oriented programs. Technical Report MSR-TR-2005-70, Microsoft Research (2005)

20. Emmi, M., Qadeer, S., Rakamarić, Z.: Delay-bounded scheduling. In: ACM SIGPLAN-SIGACT Symposium on Principles of Programming Languages (POPL), pp. 411-422 (2011)

21. Filliâtre, J.-C., Marché, C.: Multi-prover verification of C programs. In: Davies, J., Schulte, W., Barnett, M. (eds.) ICFEM 2004. LNCS, vol. 3308, pp. 15-29. Springer, Heidelberg (2004)

22. Fischer, B., Inverso, O., Parlato, G.: Cseq: A sequentialization tool for C (competition contribution). In: Piterman, N., Smolka, S.A. (eds.) TACAS 2013. LNCS, vol. 7795, pp. 616-618. Springer, Heidelberg (2013)

23. Lal, A., Qadeer, S., Lahiri, S.K.: A solver for reachability modulo theories. In: Madhusudan, P., Seshia, S.A. (eds.) CAV 2012. LNCS, vol. 7358, pp. 427-443. Springer, Heidelberg (2012)

24. Lattner, C., Adve, V.: LLVM: A compilation framework for lifelong program analysis \& transformation. In: International Symposium on Code Generation and Optimization (CGO), pp. 75-86 (2004)

25. Lattner, C., Lenharth, A., Adve, V.S.: Making context-sensitive points-to analysis with heap cloning practical for the real world. In: ACM SIGPLAN Conference on Programming Language Design and Implementation (PLDI), pp. 278-289 (2007)

26. Leino, K.R.M.: This is Boogie 2 (2008)

27. The LLVM compiler infrastructure, http://llvm.org

28. Merz, F., Falke, S., Sinz, C.: LLBMC: Bounded model checking of C and C++ programs using a compiler IR. In: Joshi, R., Müller, P., Podelski, A. (eds.) VSTTE 2012. LNCS, vol. 7152, pp. 146-161. Springer, Heidelberg (2012)

29. Păsăreanu, C.S., Mehlitz, P.C., Bushnell, D.H., Gundy-Burlet, K., Lowry, M., Person, S., Pape, M.: Combining unit-level symbolic execution and system-level concrete execution for testing NASA software. In: International Symposium on Software Testing and Analysis (ISSTA), pp. 15-26 (2008)

30. Rakamarić, Z., Hu, A.J.: Automatic inference of frame axioms using static analysis. In: IEEE/ACM International Conference on Automated Software Engineering (ASE), pp. 89-98 (2008)

31. Rakamarić, Z., Hu, A.J.: A scalable memory model for low-level code. In: Jones, N.D., Müller-Olm, M. (eds.) VMCAI 2009. LNCS, vol. 5403, pp. 290-304. Springer, Heidelberg (2009)

32. Siegel, S.F., Zirkel, T.K.: TASS: The toolkit for accurate scientific software. Mathematics in Computer Science 5(4), 395-426 (2011)

33. International competition on software verification (SV-COMP), http://sv-comp.sosy-lab.org

34. Wang, W., Barrett, C., Wies, T.: Cascade 2.0. In: McMillan, K.L., Rival, X. (eds.) VMCAI 2014. LNCS, vol. 8318, pp. 142-160. Springer, Heidelberg (2014) 